\begin{tabular}{|c|c|}
\hline Asunto & $\begin{array}{l}\text { Your Paper has now been accepted for } \\
\text { publication }\end{array}$ \\
\hline Remitente & $\begin{array}{l}\text { Journal of Physics: Condensed Matter } \\
\text { <onbehalfof@manuscriptcentral.com>, }\end{array}$ \\
\hline Destinatario & $\begin{array}{l}\text { <leandrofrisco@gmail.com>, <miraglia@iafe.uba.ar>, } \\
\text { <msilvia@iafe.uba.ar>, }\end{array}$ \\
\hline Responder a & <jpcm@iop.org>, \\
\hline Fecha & 2018-09-03 09:14 \\
\hline
\end{tabular}

Dear Dr Gravielle,

Re: "Spot-beam effect in grazing atom-surface collisions: From quantum to classical" by Frisco, Leandro; Miraglia, J; Gravielle, M

Article reference: JPCM-112119.R1

We are pleased to tell you that we have now formally accepted your Paper. We have everything we need to proceed to publish your Paper in Journal of Physics: Condensed Matter. Unless you opted out during the submission process, the accepted manuscript (http://iopscience.iop.org/page/acceptedmanuscripts) will be made available online within the next 24 hours. You will receive an email to confirm this, which will also include the permanent DOI to use to cite your work.

If you have chosen to publish your Paper on an open access basis, or if there are other charges related to your Paper you will receive an email with details on how to pay within the next few days.

We will contact you again soon when proofs of your article are ready for final approval. Please return your article proofs by the date given to enable us to publish the final version of record as soon as possible.

All articles published by IOP Publishing are available online to readers at http://iopscience.org $L$. For more information, please contact our Customer Services department at custserv@iop.org. For advice on complying with US funder requirements, please go to http://iopscience.iop.org/info/page/chorus.

Thank you for choosing to publish in Journal of Physics: Condensed Matter. We look forward to publishing your Paper.

Yours sincerely

Hector Murphy

On behalf of the IOP peer-review team:

Executive Editor - Thomas Sharp

Editor - Marric Stephens

Associate Editors - Graham Stinton, Kate Porter and Piers Stanger

Editorial Assistants - Jason Wotherspoon and Hector Murphy

Want to find out what is happening to your submission right now? Track your article here:

https://publishingsupport.iopscience.iop.org/track-my-article/?

utm source=Track\%20my \%20article\&utm medium=Email

j.pcm@iop.org

IOP Publishing

Temple Circus, Temple Way, Bristol

BS1 $6 \mathrm{HG}$, UK

www. iopscience.org $\angle j$.jpcm

2017 Impact Factor: 2.617

Letter reference: DRWA03 


\title{
Spot-beam effect in grazing atom-surface collisions: From quantum to classical
}

\author{
L. Frisco ${ }^{1}$, J.E. Miraglia ${ }^{1,2}$ and M.S. Gravielle ${ }^{2}$ \\ 1 Dpto. de Física, FCEN, Universidad de Buenos Aires, Buenos Aires, Argentina \\ 2 Instituto de Astronomía y Física del Espacio (IAFE, UBA-CONICET), Casilla \\ de Correo 67, Sucursal 28, C1428EGA Buenos Aires, Argentina \\ E-mail: msilvia@iafe.uba.ar
}

\begin{abstract}
Grazing incidence fast atom diffraction (GIFAD) is a sensitive tool for surface analysis, which strongly relies on the quantum coherence of the incident beam. In this article we study the spot-beam effect, due to contributions coming from different positions of the focus point of the incident particles, which affects the coherence of GIFAD spectra. We show that the influence of the spot-beam effect on GIFAD patterns depends on the width of the surface area that is coherently lighted by the atomic beam. While for extended illuminations the spotbeam contribution plays a minor role, when a narrow surface area is coherently lighted, the spot-beam effect allows projectiles to explore different zones of a single crystallographic channel, bringing to light intra-channel interference structures. In this last case the spot-beam effect gives also rise to a non-coherent background, which deteriorates the visibility of the interference structures. We found that by varying the impact energy, while keeping the same collimating setup, it is possible to switch gradually from quantum to classical projectile distributions. Present results are compared with available experimental data, making evident that the inclusion of focusing effects is necessary for the proper theoretical description of the experimental spectra.
\end{abstract}

Keywords: surface analysis, fast atom diffraction, coherence length, focusing effects

Submitted to: J. Phys.: Condens. Matter 


\section{Introduction}

Over the years surface analysis techniques involving collisions with atomic particles have strongly contributed to the characterization of the surface properties of solids $[1,2,3]$. Among them, grazing-incidence fast atom diffraction (GIFAD or FAD), developed in the last decade $[4,5]$, can be considered as one of the most sensitive methods to investigate the morphological and electronic characteristics of ordered surfaces [6]. GIFAD is a versatile analysis technique that can be applied to a wide variety of materials $[7,8,9,10,11,12,13]$, providing structural parameters of the topmost atomic layer with an extraordinary accuracy $[14,15,16,17,18,19]$.

The use of GIFAD as a surface analysis tool requires of both the observation of well-resolved interference structures and its appropriate theoretical interpretation. An essential aspect in GIFAD is the degree of quantum coherence of the incident beam, which governs the general shape of the interference patterns. The degree of coherence of the incident particles depends on the collimating setup and the incidence conditions. In Refs. [20, 21, 22] it was shown that for a given collision system, with fixed incidence energy and angle, the experimental collimating scheme controls the overall features of the projectile distribution, allowing one to examine two different interference mechanisms - inter-channel or intra-channel interferences - by varying the size of the collimating slit. In an equivalent way, the incidence conditions were shown to determine the mechanism that prevails in GIFAD spectra produced by using a given collimating setup [23]. Both behaviors are related to the transverse length of the surface area that is coherently lighted by the incident beam, whose knowledge becomes crucial for an appropriate comparison between experiments and simulations.

Within the frame of the above mentioned focusing effects, in this article we investigate the influence of the spot-beam effect, which is produced by randomdistributed focus points of the incident particles, on the visibility of the interference structures of GIFAD spectra. We demonstrate that when the transverse coherence length of the impinging atoms is smaller than the width of the channel, the spotbeam effect allows projectiles to probe different regions of the atom-surface potential, giving rise to intrachannel (supernumerary rainbow) maxima. In this case the spot-beam contribution introduces also a non-coherent background, which strongly modifies the visibility of the interference signatures, contributing to the transition from quantum to classical projectile distributions.

The study is confined to fast $\mathrm{He}$ and Ne atoms grazingly impinging on $\operatorname{LiF}(001)$ along the $\langle 110\rangle$ channel, for which rich diffraction patterns were observed [5, 24]. To describe the atom-surface scattering we make use of the Surface-Initial Value Representation (SIVR) approximation [25], which is a semi-quantum method that has proved to provide a successful description of experimental GIFAD patterns for different collision systems [21, 26, 27]. In this version of the SIVR approach we include the spotbeam effect, that is, the variation of the relative position of the focus point (wave-packet center) of the incident particle on the crystal surface, while the size of the coherent initial wave packet is determined from the extent of the surface region that is coherently illuminated by the atomic beam after collimation, as given in Refs. [21, 22].

The paper is organized as follows: The theoretical formalism, including the spot-beam contribution, is summarized in Sec. 2. Results for Ne projectiles under different incidence conditions are presented and discussed in Secs. 3.1 and 3.2. In particular, in Sec. 3.1 we study the dependence of the spot-beam effect on the number of coherently lighted channels, while in Sec. 3.2 the gradual quantum-classical transition of the projectile distributions is analyzed. In Sec. 3.3 helium distributions for different impact energies, with the same collimating scheme, are contrasted with available experimental data. Finally, in Sec. 4 we outline our conclusions. Atomic units (a.u.) are used unless otherwise stated.

\section{Theoretical model}

In this work we extend the previous SIVR model [25] to deal with different focus points of the incident particles. The relative position of the focus point of the beam, with respect to the surface lattice sites, it is expected to play a negligible role when the transverse coherence length of the impinging particles is longer than the width of the incidence channel. But it should gain importance as the transverse coherence length decreases. Since it is not experimentally possible to control the focus position of the incident projectiles at 


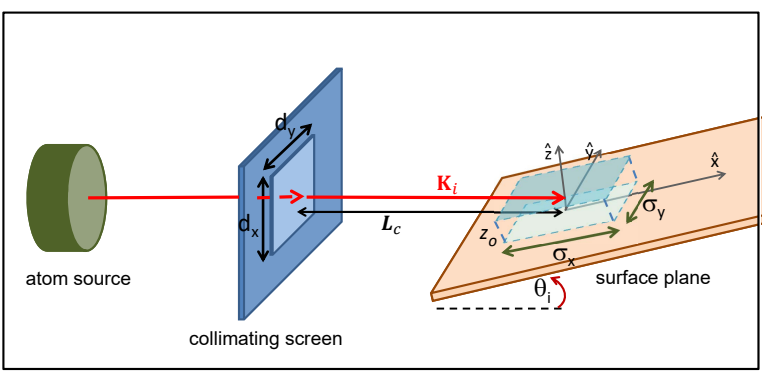

Figure 1. Depiction of the collimating scheme, together with the reference frame.

such an accuracy level, we consider that each atomic projectile impacts on the surface plane at a different position $\mathbf{R}_{s}$, which coincides with the central position of the initial coherent wave packet.

For a given position $\mathbf{R}_{s}$ of the focus point, the SIVR scattering amplitude for the elastic transition $\mathbf{K}_{i} \rightarrow \mathbf{K}_{f}, \mathbf{K}_{i}\left(\mathbf{K}_{f}\right)$ being the initial (final) momentum of the atomic projectile, with $\left|\mathbf{K}_{f}\right|=\left|\mathbf{K}_{i}\right|$, can be expressed as [25]

$$
\begin{aligned}
A_{i f}^{(S I V R)}\left(\mathbf{R}_{s}\right)= & \int d \mathbf{r}_{o} f_{i}\left(\mathbf{r}_{o}-\mathbf{R}_{s}\right) \\
& \times \int d \mathbf{k}_{o} g_{i}\left(\mathbf{k}_{o}\right) a_{i f}^{(S I V R)}\left(\mathbf{r}_{o}, \mathbf{k}_{o}\right),
\end{aligned}
$$

where $a_{i f}^{(S I V R)}\left(\mathbf{r}_{o}, \mathbf{k}_{o}\right)$ is the partial transition amplitude, given by Eq. (9) of Ref. [25], which is associated with the classical projectile path $\mathbf{r}_{t} \equiv \mathbf{r}_{t}\left(\mathbf{r}_{o}, \mathbf{k}_{o}\right)$, with $\mathbf{r}_{o}$ and $\mathbf{k}_{o}$ being the starting position and momentum, respectively, at the time $t=0$. In Eq. (1) functions $f_{i}\left(\mathbf{r}_{o}-\mathbf{R}_{s}\right)$ and $g_{i}\left(\mathbf{k}_{o}\right)$ describe the spatial and momentum profiles, respectively, of the initial coherent wave packet at a fixed distance $z_{o}$ from the surface where the atomic projectile is hardly affected by the surface interaction. Such a distance is here chosen as equal to the lattice constant.

To determine the function $f_{i}$ we assume that the atomic beam is produced by an extended incoherent quasi-monochromatic source, placed at a long distance from a rectangular collimating aperture, with sides $d_{x}$ and $d_{y}$, which is oriented perpendicular to the momentum $\mathbf{K}_{i}$ (see Fig. 1). Under the condition of extended source, given by Eqs. (A.9) and (A.10) of Ref. [22], $f_{i}\left(\mathbf{r}_{o}^{\prime}-\mathbf{R}_{s}\right)$ can be approximate by means of Gaussian functions, $G[\omega, x]=\left[2 /\left(\pi \omega^{2}\right)\right]^{1 / 4} \exp \left(-x^{2} / \omega^{2}\right)$, as $f_{i}\left(\mathbf{r}_{o}^{\prime}-\mathbf{R}_{s}\right) \simeq G\left[\sigma_{x}, x_{o}-X_{s}\right] G\left[\sigma_{y}, y_{o}-Y_{s}\right]$, where

$\sigma_{x}=\frac{L_{c} \lambda_{\perp}}{\sqrt{2} d_{x}}, \quad \sigma_{y}=\frac{L_{c} \lambda}{\sqrt{2} d_{y}}$,

denote the transverse coherence lengths of the initial coherent wave packet along the $\widehat{x}$ - and $\widehat{y}$ - directions, respectively, $\mathbf{r}_{o}^{\prime}=x_{o} \widehat{x}+y_{o} \widehat{y}$ is the component parallel to the surface plane of the starting position, and the two-dimensional vector $\mathbf{R}_{s}=X_{s} \widehat{x}+Y_{s} \widehat{y}$ corresponds to the central position of the wave packet. In Eq. (3), $L_{c}$ is the collimator-surface distance, $\lambda=2 \pi / K_{i}$ is the de Broglie wavelength of the impinging atom, and $\lambda_{\perp}=$ $\lambda / \sin \theta_{i}$ is the perpendicular wavelength associated with the initial motion normal to the surface plane, $\theta_{i}$ being the incidence angle. The momentum profile is derived from Eq. (2) by applying the Heisenberg uncertainty relation, as given by Eq. (14) of Ref. [21], leading to $g_{i}\left(\mathbf{k}_{o}\right) \simeq g_{i}\left(\Omega_{o}\right)$, with $\left|\mathbf{k}_{o}\right|=\left|\mathbf{K}_{i}\right|=\sqrt{2 m_{P} E}$ and $\Omega_{o}$ the corresponding solid angle, $m_{P}$ being the projectile mass and $E$ the total energy.

Taking into account that GIFAD patterns are produced by the interference of a single projectile with itself, contributions to the scattering probability coming from different focus points of the impinging particles must be added incoherently. Hence, the differential scattering probability in the direction of the solid angle $\Omega_{f}$ can be obtained from Eq. (1), except for a normalization factor, as

$$
\frac{d P^{(S I V R)}}{d \Omega_{f}}=\int d \mathbf{R}_{s}\left|A_{i f}^{(S I V R)}\left(\mathbf{R}_{s}\right)\right|^{2}
$$

where $\Omega_{f} \equiv\left(\theta_{f}, \varphi_{f}\right)$ is the solid angle corresponding to the $\mathbf{K}_{f}$ - direction, with $\theta_{f}$ the final polar angle, measured with respect to the surface, and $\varphi_{f}$ the azimuthal angle, measured with respect to the $\widehat{x}$ axis. In Eq. (4), the $\mathbf{R}_{s^{-}}$integral involves different relative positions within the crystal lattice, covering an area equal to a reduced unit cell of the surface.

\section{Results}

The goal of this work is to analyze the effect of the spotbeam contribution on GIFAD patterns produced under different illumination conditions of the surface [23]. For this purpose we examine final angular distributions of ${ }^{4} \mathrm{He}$ and ${ }^{20} \mathrm{Ne}$ atoms elastically scattered from $\mathrm{LiF}(001)$ along the $\langle 110\rangle$ channel, after passing through a rectangular collimating aperture situated at a distance $L_{c}=25 \mathrm{~cm}$ from the surface plane [20]. For both projectiles the surface-atom interaction was evaluated with an improved pairwise additive potential [28], which includes non-local terms of the electronic density in the kinetic, exchange and correlation energies. The potential model also takes into account projectile polarization and rumpling effects. In turn, for the numerical evaluation of the SIVR transition probability 
we employed the MonteCarlo technique to solve the sixdimensional integral involved in Eqs. (1) and (4), i.e., on $\mathbf{r}_{o}^{\prime} \equiv\left(x_{o}, y_{o}\right), \Omega_{o} \equiv\left(\theta_{o}, \varphi_{o}\right)$, and $\mathbf{R}_{s} \equiv\left(X_{s}, Y_{s}\right)$, using about $10^{7}$ points. Each of these points involves a further time integration along the classical path, included in $a_{i f}^{(S I V R)}\left(\mathbf{r}_{o}, \mathbf{k}_{o}\right)$, which was evaluated with a step-adaptive integration method [25]. Notice that in our previous SIVR calculations [25, 21, 23] we did not consider the spot-beam effect, which is equivalent to remove the $\mathbf{R}_{s^{-}}$integral from Eq. (4).

Concerning the interference structures of GIFAD spectra, it is now well-established that they come from the combination of inter- and intra- channel interferences [6], each of them being associated with a different factor of the SIVR transition amplitude [25]. The inter-channel factor, produced by interference among equivalent trajectories running along different parallel channels, gives rise to equally spaced and intense Bragg peaks, while the intra-channel factor, due to interference inside a single channel, originates supernumerary rainbow maxima [29, 6]. Accordingly, when the surface area coherently lighted by the atomic beam covers a region containing an array of parallel channels, the partial transition amplitude given by Eq. (1) displays Bragg peaks, whose intensities are modulated by the intra-channel factor. But when only one channel is coherently illuminated, $\left|A_{i f}^{(S I V R)}\left(\mathbf{R}_{s}\right)\right|$ presents supernumerary rainbow peaks, without any trace of Bragg interference, leading to a pure intrachannel interference spectrum. Thence, the number of coherently lighted channels results a critical parameter that determines not only the general shape of GIFAD distributions, but also the relative importance of the spot-beam contribution, as it will be discussed below.

\subsection{Dependence of the spot-beam effect on the number of coherently lighted channels}

The number $N$ of coherently illuminated channels can be roughly estimated from the transverse coherence length of the incident particles as

$N \simeq \frac{2 \sigma_{y}}{a_{y}}=\frac{\sqrt{2} L_{c}}{d_{y}} \frac{2 \pi}{a_{y} K_{i}}$,

where $\sigma_{y}$ is given by Eq. (3) and $a_{y}$ denotes the width of the axial channel, with $a_{y}=5.4$ a.u. for $\langle 110\rangle$ - incidence. Since for a given collimating setup, the $N$ value depends on $K_{i}$ as given by Eq. (5), along this article we study the influence of the spotbeam effect on GIFAD spectra as a function of $N$ by varying the total energy $E=K_{i}^{2} /\left(2 m_{P}\right)$, while keeping the same collimating aperture. In Secs. 3.1 and 3.2 a square collimating slit with $d_{x}=d_{y}=0.2$ $\mathrm{mm}$ is considered. In addition, as GIFAD patterns from $\mathrm{LiF}(001)$ are essentially governed by the normal

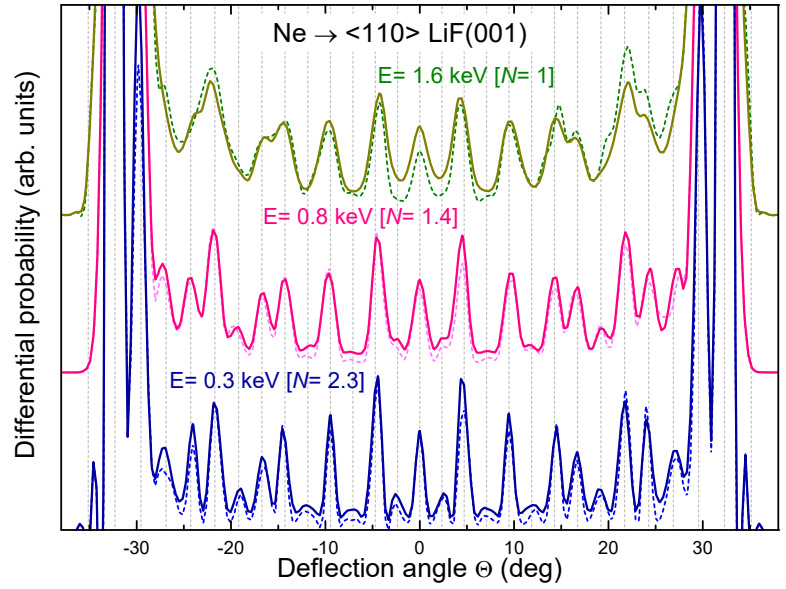

Figure 2. (Color online) Angular spectra, as a function of the deflection angle $\Theta$, for Ne atoms impinging on $\operatorname{LiF}(001)$ along the $\langle 110\rangle$ direction, with $E_{\perp}=0.3 \mathrm{eV}$ and different total energies. Solid (dashed) lines, SIVR results derived with (without) the spot-beam effect. The corresponding $N$ values, as given by Eq. (5), are also indicated. Vertical dotted lines, angular positions of Bragg peaks.

energy $E_{\perp}=E \sin ^{2} \theta_{i}[6]$, we have kept $E_{\perp}=0.3 \mathrm{eV}$ as a constant for the different impact energies.

In Fig. 2 we plot the SIVR differential probabilities, as a function of the deflection angle $\Theta=\arctan \left(\varphi_{f} / \theta_{f}\right)$, for $\mathrm{Ne}$ atoms impinging on $\mathrm{LiF}$ with three different energies: $E=0.3,0.8$, and 1.6 $\mathrm{keV}$. Under ideal scattering conditions, involving the incidence of transversely extended wave packets, these $\Theta$ - distributions were expected to be independent of $E$ at the same $E_{\perp}[30]$. Nevertheless, the spectra are strongly affected by the total energy if the same collimating setup is used, as a consequence of the variation of the $N$ value derived from Eq. (5) [23]. Therefore, in Fig. 2 the projectile distribution for $E=0.3 \mathrm{keV}$ (i.e., $N=2.3$ ) displays well defined Bragg peaks, but these Bragg structures fade out progressively as the energy increases, causing the spectrum for $E=1.6 \mathrm{keV}$ (i.e., $N=1.0$ ) to present supernumerary maxima associated with pure intrachannel interference. Furthermore, for the two lowest energies of Fig. $2-E=0.3$ and $0.8 \mathrm{keV}$ - which correspond to coherently illuminated regions with a transverse length longer than $a_{y}$, the contribution of the spot-beam effect is barely appreciable in the angular distributions. But when the width of the coherently lighted area is comparable to the channel width, as it happens for $E=1.6 \mathrm{keV}$ in Fig. 2 , differences between the spectra derived with and without the inclusion of the spot-beam effect start to be visible.

The above mentioned behavior is due to the fact that under the constraint $N \succsim 1$, the SIVR transition amplitudes corresponding to different focus points of 
the beam, given by Eq. (1), are alike, leading to

$d P^{(S I V R)} / d \Omega_{f} \simeq\left|A_{i f}^{(S I V R)}\left(\mathbf{R}_{s}=0\right)\right|^{2}$,

where $\mathbf{R}_{s}=0$ indicates a focus point situated just in the middle of the incidence channel, here named central focus point. However, when the impact energy augments beyond the limit of pure intra-channel interference, and consequently, the coherently lighted area shrinks, covering a surface region narrower than $a_{y}$, the different $Y_{s}$ coordinates of the focus points give rise to dissimilar partial projectile distributions $\left|A_{i f}^{(S I V R)}\left(\mathbf{R}_{s}\right)\right|^{2}$. Each of these partial distributions probes a different zone of the atom-surface potential within the channel, causing the contribution of the spot-beam effect, associated with the $\mathbf{R}_{s}$-integral in Eq. (4), to become important.

In order to study thoroughly the $N$ - dependence of the spot-beam effect for narrow illuminations, in Fig. 3 we show neon projectile distributions for higher impact energies - $E=2,3$, and $8 \mathrm{keV}$ - which correspond to $N=0.9,0.7$, and 0.4 , respectively. In all the panels, results derived from Eq. (4), including the spot-beam contribution, are contrasted with those obtained by considering only pure intra-channel interference, as given by Eq. (6) for $N=1$. From Fig. 3 we found that the spot-beam contribution keeps the angular positions of supernumerary maxima, but introduces a non-coherent background in the central region of the spectrum, around the direction of specular reflection (i.e., $\Theta \simeq 0$ ), in relation to that for single-channel illumination. The angular extension of such a spotbeam background is sensitive to $N$, increasing as $N$ diminishes, as observed by comparing Figs. 3 (a) and (b).

The role played by the spot-beam effect is even more relevant when the transverse length of the surface area that is coherently illuminated by the beam is about or smaller than the half width of the incidence channel. In Fig. 3 (c) the projectile distribution for $8 \mathrm{keV} \mathrm{Ne}$ atoms (i.e., $N=0.4$ ) is severely affected by the spot-beam effect when it is contrasted with that due to pure intra-channel interference, corresponding to $N=1$. Different $\mathbf{R}_{s}$ positions allow projectiles to separately explore zones of the potential energy surface with positive or negative slope, producing interference structures placed at negative or positive deflection angles, respectively. Only when these partial contributions are added, as given by Eq. (4), the angular spectrum including the spotbeam contribution presents defined supernumerary peaks in the whole angular range. But in this case the spot-beam effect gives also rise to a wide noncoherent background, which reduces the visibility of the interference patterns, in comparison with that of the pure intra-channel spectrum, as it will be discussed in the next Section.

\subsection{Focusing effect in the transition from quantum to classical distributions}

In this Section we investigate how the decreasing of $N$ below the unit reduces the visibility of the diffraction patterns, leading to the gradual switch from quantum projectile distributions, containing intrachannel interference structures, to classical spectra without signatures of interference. With this aim it is convenient to analyze the profile of the atom-surface potential near the reflection region of projectiles, which governs the intra-channel interference in a first approach. Beforehand, we stress that our SIVR calculations were obtained from a three-dimensional atom-surface potential and no dimension reduction was made during the dynamics. However, since GIFAD patterns are essentially sensitive to the averaged potential energy surfaces along the incidence channel, such effective equipotential contours can provide us useful insights of the intra-channel interference mechanism.

For Ne atoms impinging on $\operatorname{LiF}(001)$ along the $\langle 110\rangle$ direction, in Fig. 4 (a) we plot the averaged equipotential curve - $z(y)$ - corresponding to $E_{\perp}=0.3$ $\mathrm{eV}$, as a function of the coordinate $y$ across the channel, normalized by the width $a_{y}$. Within this simplified picture, the intra-channel interference is produced by the coherent addition of transition amplitudes $a_{i f}^{(S I V R)}\left(\mathbf{r}_{o}, \mathbf{k}_{o}\right)$ corresponding to trajectories reflecting at turning points with different $y$ coordinates inside the channel, but with the same slope $d z / d y$ of the averaged equipotential curve, which determines the final azimuthal angle [6]. For the present case, from Fig. 4 (b) it is observed that there are only two different trajectories that contribute to the intrachannel interference pattern at a given angular position $\varphi_{f}$, except around rainbow angles where several (infinite) turning points coalesce at a maximum or minimum of $d z / d y$. Then, in GIFAD distributions an essential requirement to observe a supernumerary rainbow structure at a given $\varphi_{f}$ or $\Theta$ angle (inside the angular range defined by the rainbow peaks) is given by the condition that the $N$ value must be longer than $l_{\mathrm{t}}=$ $l_{y} / a_{y}$, where $l_{y}$ denotes the transverse distance between the turning points of the corresponding interfering trajectories. This fact is illustrated in Fig. 5, where the distribution for $\langle 110\rangle$ incidence of $16 \mathrm{keV} \mathrm{Ne}$ atoms, corresponding to $N=0.3$, is displayed. In this case the spectrum obtained including the spotbeam effect does not show the central interference maximum, associated with the highest $l_{\mathrm{t}}$ value (i.e., $l_{\mathrm{t}}=0.5$ corresponding to two trajectories on top of the $\mathrm{Li}^{+}$- and $\mathrm{F}^{-}$- crystallographic rows, respectively), 


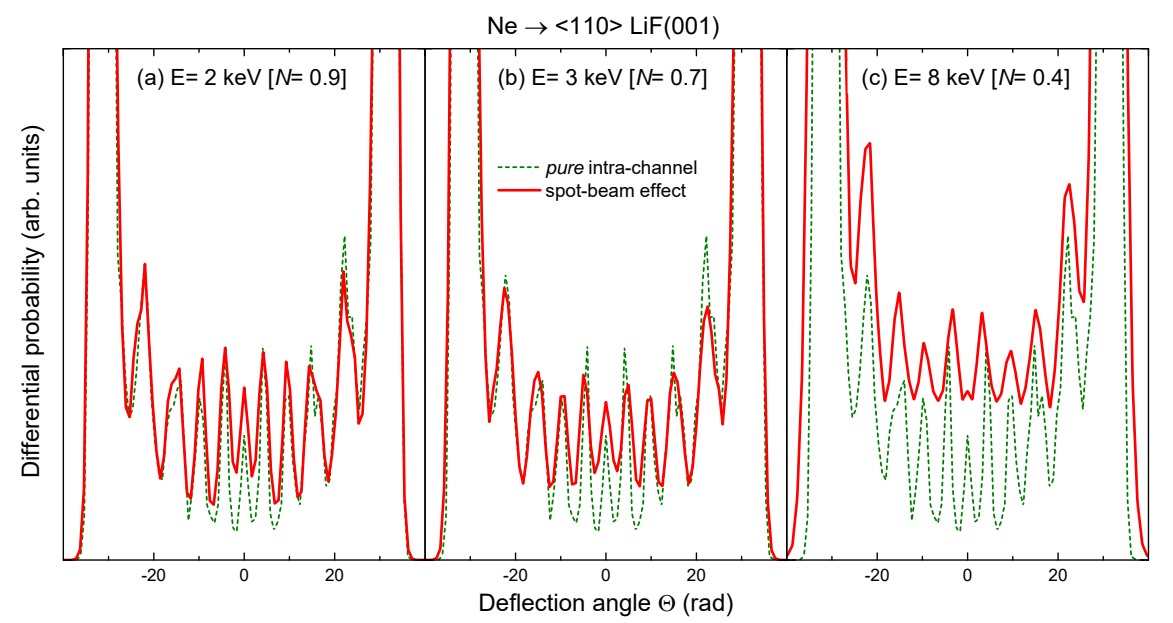

Figure 3. (Color online) Angular spectra, as a function of the deflection angle $\Theta$, for Ne atoms along the $\langle 110\rangle$ direction, with $E_{\perp}=0.3 \mathrm{eV}$. Results for (a) $E=2 \mathrm{keV}[N=0.9]$, (b) $E=3 \mathrm{keV}[N=0.7]$, and (c) $E=8 \mathrm{keV}[N=0.4]$ are displayed. Red solid line, angular distribution including the spot-beam effect, as given by Eq. (4); dark-green dashed line, pure intra-channel distribution corresponding to $N=1$, given by Eq. (6).

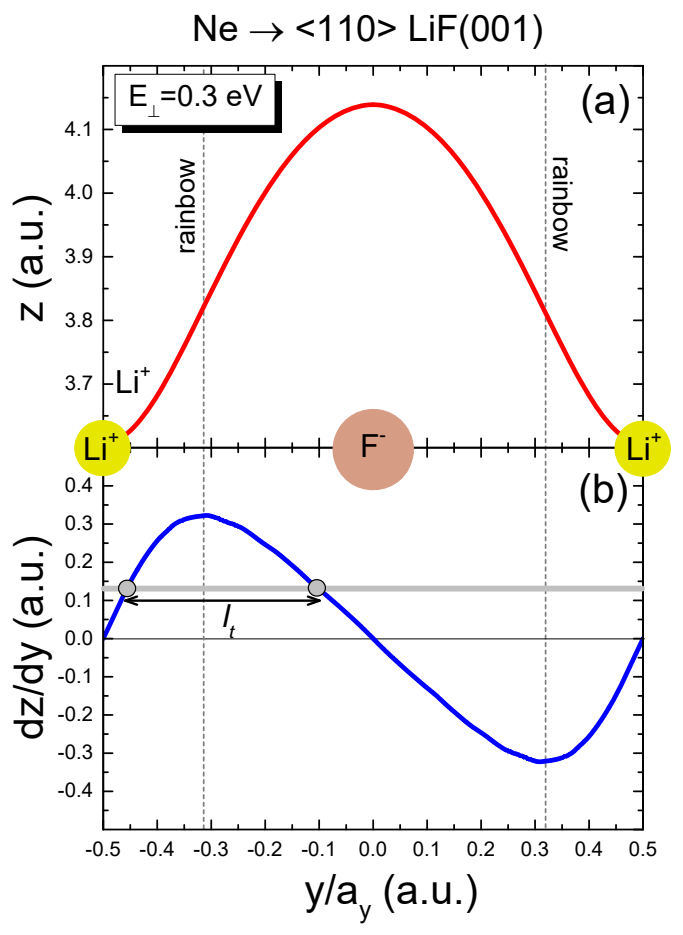

Figure 4. (Color online) Analysis of the equipotential contour, averaged along the $\langle 110\rangle$ channel, for the Ne-LiF(001) interaction. (a) Red solid line, equipotential curve $z(y)$ for $E_{\perp}=0.3 \mathrm{eV}$; (b) derivative $d z / d y$ of the equipotential curve of (a). Gray circles, turning point positions corresponding to two different trajectories that interfere at a given deflection angle; vertical dashed lines indicate turning point positions corresponding to trajectories that contribute to the rainbow maxima.

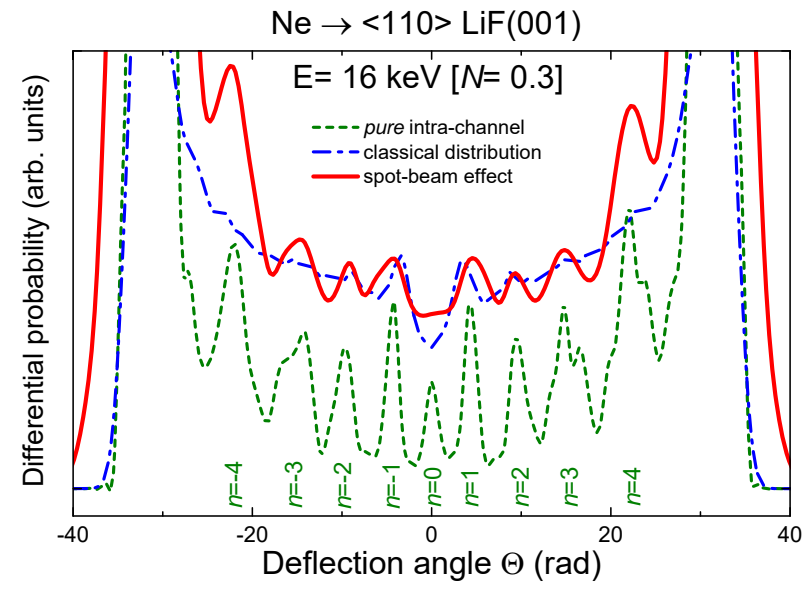

Figure 5. (Color online) Analogous to Fig. 3 for $E=16 \mathrm{keV}$ $[N=0.3]$. Blue dot-dashed line, classical projectile distribution for $N=1$. The $n$ values indicate different supernumerary rainbow peaks.

and supernumerary rainbow peaks are visible at larger deflection angles only. In addition, the visibility of the interference structures diminishes and the spectrum tends to the classical distribution, which displays only pronounced rainbow maxima.

The quantum-classical transition of GIFAD distributions can be quantitatively studied by analyzing the visibility $\mathcal{V}(n)$ associated with the supernumerary rainbow maximum labelled with $n$ in Fig. 5, where $n=0, \pm 1, \pm 2, . ., n=0$ corresponding to the central peak [31]. Like in optics [32], we define the visibility in GIFAD as

$\mathcal{V}(n)=\frac{I_{\max }^{(n)}-I_{\min }^{(n)}}{I_{\max }^{(n)}+I_{\min }^{(n)}}$, 


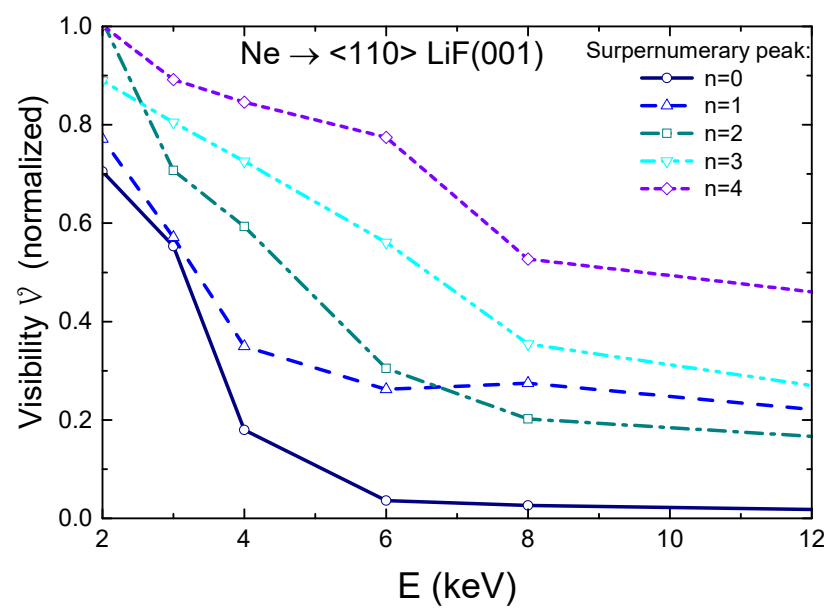

Figure 6. (Color online) Visibility $\mathcal{V}(n)$ (normalized to that for $N=1$ ), as a function of $E$, for Ne projectiles colliding along $\langle 110\rangle$ with $E_{\perp}=0.3 \mathrm{eV}$.

where $I_{\max }^{(n)}$ is the differential probability $d P^{(S I V R)} / d \Theta$, derived from Eq. (4), at the $n$ - supernumerary rainbow maximum, and $I_{\min }^{(n)}$ denotes the averaged value of the differential probability at the positions of the two adjacent minima. This visibility provides a measure of the degree of coherence of the atomic beam $[32,33]$. In Fig. 6 we show $\mathcal{V}(n)$, normalized to that for $N=1$, as a function of the impact energy, for $\mathrm{Ne}$ projectiles colliding along $\langle 110\rangle$ with $E_{\perp}=0.3 \mathrm{eV}$. As a consequence of the spot-beam effect, under the same collimating conditions the visibility tends to decrease when the energy increases beyond the energy limit of pure intra-channel interference. Such a decreasing is more steeply for the central peaks than for the outermost ones, in accord with the condition $N \succsim l_{\mathrm{t}}$ for the observation of supernumerary maxima. For higher $E$ (lower $N$ ) values, the interference structures gradually blur out and all $\mathcal{V}(n)$ slowly decrease, making projectile distributions reach the classical limit, i.e., $\mathcal{V}(n) \approx 0$, where all the quantum signatures disappear.

\subsection{Experimental comparison}

To test the predicted influence of the incidence conditions, in Fig. 7 simulations derived from Eq. (4) are compared with available experimental distributions [34] for helium atoms impinging on $\operatorname{LiF}(001)$ along the $\langle 110\rangle$ channel. These two-dimensional angular distributions were obtained by varying the impact energy but keeping a fixed incidence angle, i.e., $\theta_{i}=$ $1.1 \mathrm{deg}$ [34]. In order to reproduce the experiments, in this Section we have considered a rectangular slit with sides $d_{x}=1.5 \mathrm{~mm}$ and $d_{y}=0.3 \mathrm{~mm}$, which produces an angular dispersion $\omega_{\varphi}=0.05 \mathrm{deg}$, comparable to the experimental value [34]. In Fig.
7, for $E=1.25 \mathrm{keV}$ (top panels) the theoretical distribution is in accord with the experimental one, showing not fully resolved Bragg peaks associated with $N=1.6$. Instead, for $E=3.50 \mathrm{keV}$ (middle panels) the Bragg peaks completely disappear and the simulated and experimental GIFAD patterns display only supernumerary maxima corresponding to a singlechannel illumination. Lastly, for $E=9.00 \mathrm{keV}$ $[N=0.6]$ (lower panels) the interference maxima are barely visible as isolated peaks in the simulated angular spectrum due to the contribution of the spotbeam effect. In this case, both the theoretical and the experimental distributions tend to the classical one, showing a broad high intensity contribution at $\varphi_{f}=0$ and two intense rainbow peaks at the outermost angles.

Therefore, the reasonable good agreement between theory and experiment observed in Fig. 7 strongly suggests that the energy dependence of the general features of experimental GIFAD distributions is mainly produced by the variation of the transverse coherence length, as it was proposed in Ref. [34]. However, at this point it is necessary to mention that there are other effects not included in our model, like inelastic processes $[35,36,37]$, which can contribute to reduce the coherence, promoting to the transition from quantum to classical projectile distributions. Moreover, notice that our simulations do not include thermal vibrations of lattice atoms [28] and the results were not convoluted with the detector resolution, both effects which are expected to smooth the theoretical spectra. Regarding thermal effects, the experiments of Ref. [34] were carried out with the crystal surface at room temperature [14]. At such a surface temperature, the thermal fluctuations of the LiF crystal are expected to affect more the intensity of the external peaks than those corresponding to the internal maxima [28], indicating that the spot-beam effect dominates over temperature-induced decoherence of the internal supernumerary peaks.

\section{Conclusions}

We have investigated the influence of the spot-beam effect, originated by random positions of the focus point of the beam, on the general characteristics of GIFAD patterns produced from a $\mathrm{LiF}(001)$ surface. The relevance of the spot-beam contribution was analyzed in terms of the number $N$ of equivalent parallel channels that are coherently illuminated by the atomic beam. We have shown that when several parallel channels are coherently lighted, the spot-beam effect does not significantly affect the GIFAD patterns. But it becomes important when only a portion of a single crystallographic channel is coherently lighted by the impinging particles. In particular, for $N$ 


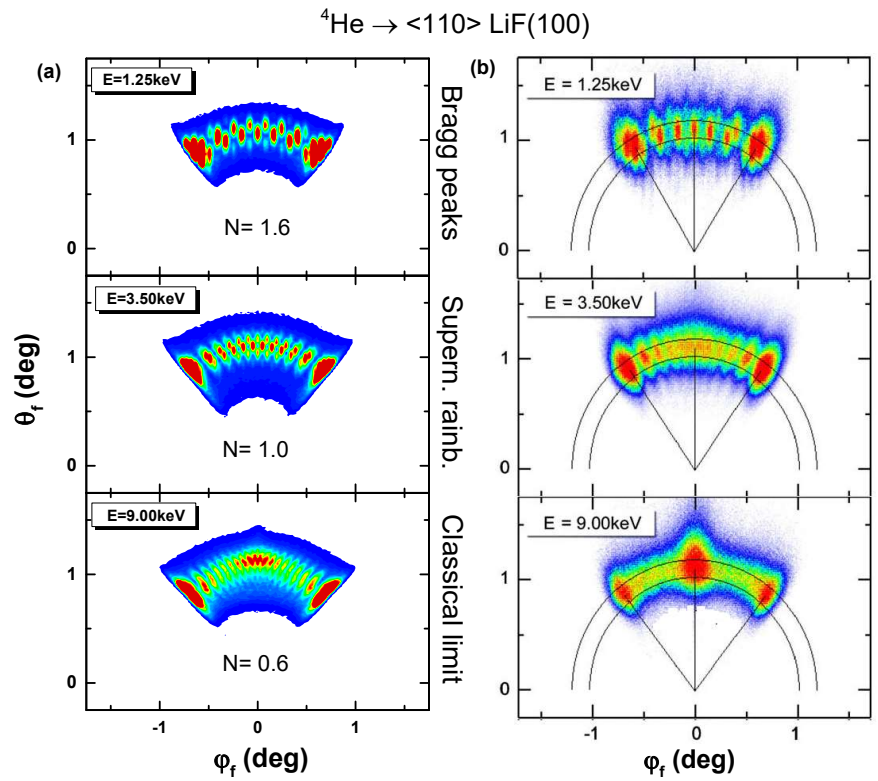

Figure 7. (Color online) Two-dimensional angular distributions, as a function of $\theta_{f}$ and $\varphi_{f}$, for He atoms impinging on LiF(001) along $\langle 110\rangle$ with $\theta_{i}=1.1 \mathrm{deg}$. (a) (left panels) Simulated SIVR results derived by considering a collimating slit of width $d_{y}=0.3$ $\mathrm{mm}$. (b) (right panels) Experimental distributions extracted from Ref. [34]. In both columns, different impact energies - $E=1.25$, 3.50 , and $9.00 \mathrm{keV}$ - are considered. The corresponding $N$ values, as given by Eq. (5), are indicated.

values in the range $0.4 \precsim N \precsim 1$ the spotbeam effect helps to recover supernumerary rainbow maxima that probe different regions of the atomsurface potential. In addition, we found that for $N \precsim 1$ the spot-beam contribution gives rise to a non-coherent background, which modifies strongly the visibility of the interference structures. Consequently, the spotbeam effect contributes to the gradual quantumclassical transition of the projectile distributions when the impact energy augments under fixed collimating conditions.

\section{Acknowledgments}

This work was carried out with financial support from CONICET, UBA and ANPCyT of Argentina.

\section{References}

[1] K. Heinz, U. Starke, and J. Bernhardt. Surface holography with LEED electrons. Prog. Surf. Sci., 64:163-178, 2000.

[2] H. Niehus, W. Heiland, and E. Taglauer. Low-energy ion scattering at surfaces. Surf. Sci. Rep., 17:213-303, 1993.

[3] D. Farias and K-H. Rieder. Atomic beam diffraction from solid surfaces. Rep. Prog. Phys., 61:1575-1664, 1998.

[4] A. Schüller, S. Wethekam, and H. Winter. Diffraction of Fast Atomic Projectiles during Grazing Scattering from a LiF(001) Surface. Phys. Rev. Lett., 98:016103, 2007.

[5] P. Rousseau, H. Khemliche, A. G. Borisov, and P. Roncin. Quantum Scattering of Fast Atoms and Molecules on Surfaces. Phys. Rev. Lett., 98:016104, 2007.
[6] H. Winter and A. Schüller. Fast atom diffraction during grazing scattering from surfaces. Prog. Surf. Sci., 86:169-221, 2011.

[7] M. Debiossac, A. Zugarramurdi, H. Khemliche, P. Roncin, A. G. Borisov, A. Momeni, P. Atkinson, M. Eddrief, F. Finocchi, and V. H. Etgens. Combined experimental and theoretical study of fast atom diffraction on the $\beta_{2}(2 \times 4)$ reconstructed GaAs(001) surface. Phys. Rev. $B, 90: 155308,2014$.

[8] M. Busch, A. Schüller, S. Wethekam, and H. Winter. Fast Atom Diffraction at Metal Surface. Surf. Sci., 603:L23L26, 2009.

[9] H. Winter, J. Seifert, D. Blauth, M. Busch, A. Schüller, and S. Wethekam. Structure of ultrathin oxide layers on metal surfaces from grazing scattering of fast atoms. Appl. Surf. Sci., 256:365-370, 2009.

[10] A. Schüller, M. Busch, S. Wethekam, and H. Winter. Fast Atom Diffraction from Superstructures on a $\mathrm{Fe}(110)$ Surface. Phys. Rev. Lett., 102:017602, 2009.

[11] J. Seifert, M. Busch, E. Meyer, and H. Winter. Surface Structure of Alanine on $\mathrm{Cu}(110)$ Studied by Fast Atom Diffraction. Phys. Rev. Lett., 111:137601, 2013.

[12] A. Zugarramurdi, M. Debiossac, P. Lunca-Popa, A. J. Mayne, A. Momeni, A. G. Borisov, Z. Mu, P. Roncin, and H. Khemliche. Determination of the geometric corrugation of graphene on $\mathrm{SiC}(0001)$ by grazing incidence fast atom diffraction. Appl. Phys. Lett., 106:101902, 2015.

[13] A. Momeni, E. M. Staicu Casagrande, A. Dechaux, and H. Khemliche. Ultrafast Crystallization Dynamics at an Organic-Inorganic Interface Revealed in Real Time by Grazing Incidence Fast Atom Diffraction. J. Phys. Chem. Lett., 9:908-913, 2018.

[14] A. Schüller, S. Wethekam, D. Blauth, H. Winter, F. Aigner, N. Simonović, B. Solleder, J. Burgdörfer, and L. Wirtz. Rumpling of $\mathrm{LiF}(001)$ surface from fast atom diffraction. Phys. Rev. A, 82:062902, 2010. 
[15] A. Schüller, D. Blauth, J. Seifert, M. Busch, H. Winter, K. Gärtner, R. Włodarczyk, J. Sauer, and M. Sierka. Fast atom diffraction during grazing scattering from a $\mathrm{MgO}(001)$ surface. Surf. Sci., 606:161-173, 2012.

[16] B. Lalmi, H. Khemliche, A. Momeni, P. Soulisse, and P. Roncin. High resolution imaging of superficial mosaicity in single crystals using grazing incidence fast atom diffraction. J. Phys.: Condens. Matter, 24:442002, 2012.

[17] J. Seifert and H. Winter. Quantitative structure determination using grazing scattering of fast atoms: Oxygeninduced missing-row reconstruction of $\mathrm{Mo}(112)$. Phys. Rev. B, 93:205417, 2016.

[18] M. del Cueto, A. S. Muzas, M. F. Somers, G. J. Kroes, C. Díaz, and F. Martín. Exploring surface landscapes with molecules: rotatonally induced diffraction of $\mathrm{H}_{2}$ on $\mathrm{LiF}(001)$ under fast grazing incidence conditions. Phys. Chem. Chem. Phys., 19:16317-16322, 2017.

[19] M. Debiossac, P. Atkinson, A. Zugarramurdi, M. Eddrief, F. Finocchi, V. H. Etgens, A. Momeni, H. Khemliche, A. G. Borisov, and P. Roncin. Fast atom diffraction inside a molecular beam epitaxy chamber, a rich combination. Appl. Surf. Sci., 391:53-58, 2017.

[20] J. Seifert, J. Lienemann, A. Schüller, and H. Winter. Studies on coherence and decoherence in Fast Atom Diffraction. Nucl. Instrum. Methods Phys. Res. B, 350:99-105, 2015.

[21] M. S. Gravielle and J. E. Miraglia. Influence of beam collimation on fast-atom diffraction studied via a semiquantum approach. Phys. Rev. A, 92:062709, 2015.

[22] M. S. Gravielle and J. E. Miraglia. Single- and doubleslit collimating effects on fast-atom diffraction spectra. Nucl. Instrum. Methods Phys. Res. B, 382:42-48, 2016.

[23] M. S. Gravielle. Fast interaction of atoms with crystal surfaces: coherent lighting. J. Phys. Conf. Ser., 875:012006, 2017.

[24] M. S. Gravielle, A. Schüller, H. Winter, and J.E. Miraglia. Fast atom diffraction for grazing scattering of Ne atoms from a $\operatorname{LiF}(001)$ surface. Nucl. Instr. Methods Phys. Res. $B, 269: 1208-1211,2011$.

[25] M. S. Gravielle and J. E. Miraglia. Semiquantum approach for fast atom diffraction: Solving the rainbow divergence. Phys. Rev. A, 90:052718, 2014.

[26] G. A. Bocan, J. D. Fuhr, and M. S. Gravielle. van derWaals effects on grazing-incidence fast-atom diffraction for $\mathrm{H}$ on LiF(001). Phys. Rev. A, 94:022711, 2016.

[27] G. A. Bocan and M. S. Gravielle. GIFAD for $\mathrm{He} / \mathrm{KCl}(001)$. Structure in the pattern for $\langle 110\rangle$ incidence as a measure of the projectile-cation interaction. Nucl. Instr. Methods Phys. Res. B, 421:1-6, 2018.

[28] J. E. Miraglia and M. S. Gravielle. Reexamination of the interaction of atoms with a $\mathrm{LiF}(001)$ surface. Phys. Rev. A, 95:022710, 2017.

[29] A. Schüller and H. Winter. Supernumerary Rainbows in the Angular Distribution of Scattered Projectiles for Grazing Collisions of Fast Atoms with a $\mathrm{LiF}(001)$ Surface. Phys. Rev. Lett., 100:097602, 2008.

[30] A. Schüller, H. Winter, M. S. Gravielle, J. M. Pruneda, and J. E. Miraglia. He-LiF surface interaction potential from fast atom diffraction. Phys. Rev. A, 80:062903, 2009.

[31] Notice that the parameter $n$ used to label the different supernumerary maxima does not coincide with the supernumerary rainbow order as defined in ref. [6].

[32] M. Born and E. Wolf. Principles of Optics, chapter 10. Pergamon Press, Oxford, 1986.

[33] C. S. Adams, M. Sigel, and J. Mlynek. Atom optics. Phys. Rep., 240:143-210, 1994.

[34] A. Schüller and H. Winter. Difraction of fast atoms under axial surface channeling conditions. Nucl. Instr. Methods Phys. Res. B, 267:628-633, 2009.
[35] A. Al Taleb, G. Anemone, W. W. Hayes, J. R. Manson, and D. Farías. Mutiphonon excitation and quantum decoherence in neon scattering from solid surfaces. Phys. Rev. B, 95:075414, 2017.

[36] G. Anemone, A. Al Taleb, W. W. Hayes, J. R. Manson, and D. Farías. Quantum Decoherence Behavior in Neon Scattering from $\mathrm{Ru}(0001)$ and Graphene/Ru(0001) Surfaces: Experiment and Comparison with Calculations. J. Phys. Chem. C, 121:22815-22825, 2017.

[37] P. Roncin and M. Debiossac. Elastic and inelastic diffraction of fast atoms, Debye-Waller factor, and MössbauerLamb-Dicke regime. Phys. Rev. B, 96:035415, 2017. 